\title{
D2-40, a novel immunohistochemical marker in differentiating dermatofibroma from dermatofibrosarcoma protuberans
}

\author{
Bizhan Bandarchi ${ }^{1}$, Linglei Ma ${ }^{2,3}$, Celia Marginean ${ }^{4}$, Sara Hafezi ${ }^{1}$, Judit Zubovits ${ }^{1}$ and \\ Golnar Rasty ${ }^{1}$ \\ ${ }^{1}$ Department of Anatomic Pathology, Sunnybrook Health Sciences Center, University of Toronto, Toronto, \\ ON, Canada; ${ }^{2}$ Department of Pathology, University of Michigan Medical Center, Ann Arbor, MI, USA; \\ ${ }^{3}$ Department of Dermatology, University of Michigan Medical Center, Ann Arbor, MI, USA and ${ }^{4}$ Department \\ of Pathology, Ottawa Hospital, University of Ottawa, Ottawa, ON, Canada
}

\begin{abstract}
The distinction between dermatofibroma, particularly cellular variant, and dermatofibrosarcoma protuberans in excisional biopsies is usually straightforward. However, a separation between the two may be sometimes challenging, especially in superficial biopsies. Although factor XIIla and CD34 immunostains are useful in differentiating dermatofibroma and dermatofibrosarcoma protuberans in most instances, focal CD34 positivity may be seen in cellular fibrous histiocytoma. Some cases reveal overlapping immunostain results. D2-40 identifies a $40-\mathrm{kDa}$ O-linked sialoglycoprotein present on a variety of tissues including testicular germ cell tumors as well as lymphatic endothelium. In this study, we investigated the utility of D2-40 in separating dermatofibroma from dermatofibrosarcoma protuberans and compared the results with other commonly used immunostains. Fifty-six cases of dermatofibroma (including six cellular variant) and 29 cases of dermatofibrosarcoma protuberans were retrieved from the archives of Department of Anatomic Pathology at Sunnybrook Health Sciences Center in University of Toronto. We applied factor XIIla, CD34, and monoclonal mouse antiD2-40 immunostains to formalin-fixed, paraffin-embedded tissue sections. All $56(100 \%)$ cases of dermatofibroma demonstrated strong and diffuse immunoreactivity to D2-40 in the spindle cells and stroma. Similarly, factor XIIla showed strong and diffuse positivity in the spindle cells. Nearly all dermatofibromas were negative for CD34 except one case revealing focal positivity. None of dermatofibrosarcoma protuberans cases were labeled by D2-40, although four cases showed weak and patchy background staining in contrary to diffuse, strong, and crisp staining seen in dermatofibromas. Our results indicate that D2-40 seems to be a sensitive immunohistochemical marker for dermatofibromas, including cellular variant. Focal and faint D2-40 staining may be seen in the stroma of dermatofibrosarcoma protuberans. Our findings suggest that D2-40 can be used as a complementary immunostain to factor XIIla and CD34 in problematic and challenging cases on superficial biopsies.

Modern Pathology (2010) 23, 434-438; doi:10.1038/modpathol.2009.176; published online 8 January 2010
\end{abstract}

Keywords: D2-40; dermatofibroma; dermatofibrosarcoma protuberans

The differential diagnoses of cutaneous spindle cell proliferation are broad and include spindle cell melanoma, spindle cell squamous cell carcinoma, fibrous and fibrohistiocytic tumors (such as derma-

Correspondence: Dr B Bandarchi, MD, Department of Anatomic Pathology, Sunnybrook Health Sciences Center, 7 Yorkview Drive, Toronto, ON, Canada M2N 2R9.

E-mail: bizhanb@yahoo.com

Received 7 August 2009; revised 13 November 2009; accepted 18 November 2009; published online 8 January 2010 tofibroma and dermatofibrosarcoma protuberans), spindle cell vascular, adipocytic, or neural proliferations. Some of these entities are rare and some are very common. The majority of these cases are straightforward on hematoxylin and eosin stains, even without utilization of immunohistochemistry study. Differentiating between dermatofibroma and dermatofibrosarcoma protuberans usually is not challenging when we are dealing with excisional biopsies. In the minority of cases, especially in superficial biopsies, the distinction 
between dermatofibroma and dermatofibrosarcoma protuberans, particularly deep cellular fibrous histiocytoma may become problematic and challenging.

Dermatofibromas are characterized by poorly demarcated dermal spindle proliferation, which is composed of admixture of collagenous stroma, histiocytes, multinucleated giant cells, and blood vessels in various proportions. Dermatofibromas also reveal overlying epidermal hyperplasia, basal layer hyperpigmentation, Grenz zone, and characteristic wrapping around the pre-existing collagen fibers at the periphery of the lesion. Cellular fibrous histiocytoma may show prominent storiform pattern and often extend into the superficial subcutaneous tissue, mimicking dermatofibrosarcoma protuberans. ${ }^{1-4}$ It may recur locally if incompletely excised. ${ }^{1}$

Dermatofibrosarcoma protuberans is characterized by diffuse irregular infiltration of subcutis (lace-like/ Swiss cheese/honeycomb pattern) by fairly uniform spindle cells with storiform pattern being common. There is often a thin Grenz zone present; however, extension into epidermis with ulceration sometimes happens. ${ }^{5}$ Despite the above-mentioned characteristic features, dermatofibroma and dermatofibrosarcoma protuberans may show overlapping features (eg indeterminate fibrohistiocytic lesion of the skin). ${ }^{6}$ These overlapping features are sometimes problematic; ie cellular spindle cell proliferations in superficial biopsies devoid of the junction of dermis and subcutis. In these instances, CD34 and factor XIIIa immunostains are often very helpful. Dermatofibroma, including cellular variants, usually reveal factor XIIIa immunoreactivity in both spindle cells and macrophages ${ }^{7}$ and dermatofibrosarcoma protuberans shows strong CD34 positivity in spindle cells. $^{8-11}$ Reportedly, CD34 and factor XIIIa immunohistochemical stains may show some overlap; ${ }^{12}$ for instance, cellular fibrous histiocytoma may show focal CD34 immunoreactivity, particularly at the periphery; indeterminate fibrohistiocytic lesion of the skin may reveal dual population of CD34 and factor XIIIa positive cells. ${ }^{6}$ In one study, 95\% dermatofibromas revealed factor XIIIa positivity, whereas $25 \%$ showed CD34 reactivity. ${ }^{13}$ In contrast, dermatofibrosarcoma protuberans were positive for CD34 and factor XIIIa in 80 and $15 \%$ of cases, respectively. ${ }^{13}$ In this study, we investigated the application of D2-40 on dermatofibroma and dermatofibrosarcoma protuberans and compared the results with findings on $\mathrm{H} \& \mathrm{E}$ stain, CD34, and factor XIIIa immunostains.

\section{Materials and methods}

Fifty-six cases of dermatofibroma (including 6 cellular fibrous histiocytoma) and 29 cases of dermatofibrosarcoma protuberance were retrieved between 2000 and 2007 from the archives of
Department of Anatomic Pathology at Sunnybrook Health Sciences Center in University of Toronto, Canada. Factor XIIIa, CD34, and monoclonal mouse anti-human D2-40 were applied on formalin-fixed, paraffin-embedded tissue sections as described earlier. ${ }^{13,14}$

\section{Results}

All $56 \quad(100 \%)$ dermatofibromas revealed strong, diffuse, and crisp cytoplasmic D2-40 positivity in the spindle cells with a granular pattern (Figure 1a-e). All 29 cases of dermatofibrosarcoma protuberans were negative for D2-40 immunostain (Figure 2a and b); however, four cases of dermatofibrosarcoma protuberans showed only focal and faint staining in the background, in contrary to diffuse, strong, granular, and sharp staining seen in dermatofibroma. All cases $(56 / 56,100 \%)$ of dermatofibroma, including cellular variants, revealed diffuse factor XIIIa positivity in the spindle cells. Nearly all cases $(55 / 56)$ of dermatofibroma were negative for CD34. One case of dermatofibroma (a cellular fibrous histiocytoma) showed focal CD34 positivity; interestingly, this focus was negative for D2-40 and factor XIIIa (mirror image). All $29(100 \%)$ cases of dermatofibrosarcoma protuberans displayed strong and diffuse immunoreactivity for CD34. All cases $(100 \%)$ of dermatofibrosarcoma protuberans were negative for factor XIIIa.

\section{Discussion}

D2-40 (IgG1) is a monoclonal antibody to M2A antigen, a 40-kDa O-linked sialoglycoprotein. ${ }^{14} \mathrm{M} 2 \mathrm{~A}$ antigen was purified by sequential lectin and antibody affinity chromatography and characterized as a monomeric surface sialoglycoprotein, extensively glycosylated with O-linked carbohydrate structures, but devoid of N-linked sugars. ${ }^{14}$ D2-40 antibody specifically detects a fixation resistant epitope on podoplanin, which is a mucin-type transmembrane glycoprotein highly expressed in lymphatic endothelium. ${ }^{15,16} \mathrm{D} 2-40$ is specific for normal lymphatic endothelium, and many vascular tumors, including Kaposi's sarcomas, lymphangiomas, a subset of angiosarcomas with partial lymphatic differentiation, hemangioblastomas, Kaposiform hemangioendotheliomas, and a subset of other vascular tumors. ${ }^{16-21} \mathrm{D} 2-40$ is also expressed in other normal and tumoral non-endothelial cells, including myoepithelial cells in breast, interstitial cells of Cajal, periphery (peripheral/basal cells) of normal sebaceous glands, non-neoplastic and neoplastic adrenal cortex, follicular dendritic cells and tumors, malignant mesothelioma (epithelioid type), pure seminomas, and seminomatous component of mixed germ cell tumors. ${ }^{22-27}$ Schacht et $a l^{27}$ also demonstrated the expression of D2-40 in some other nonendothelial cells beyond the above-mentioned cells 

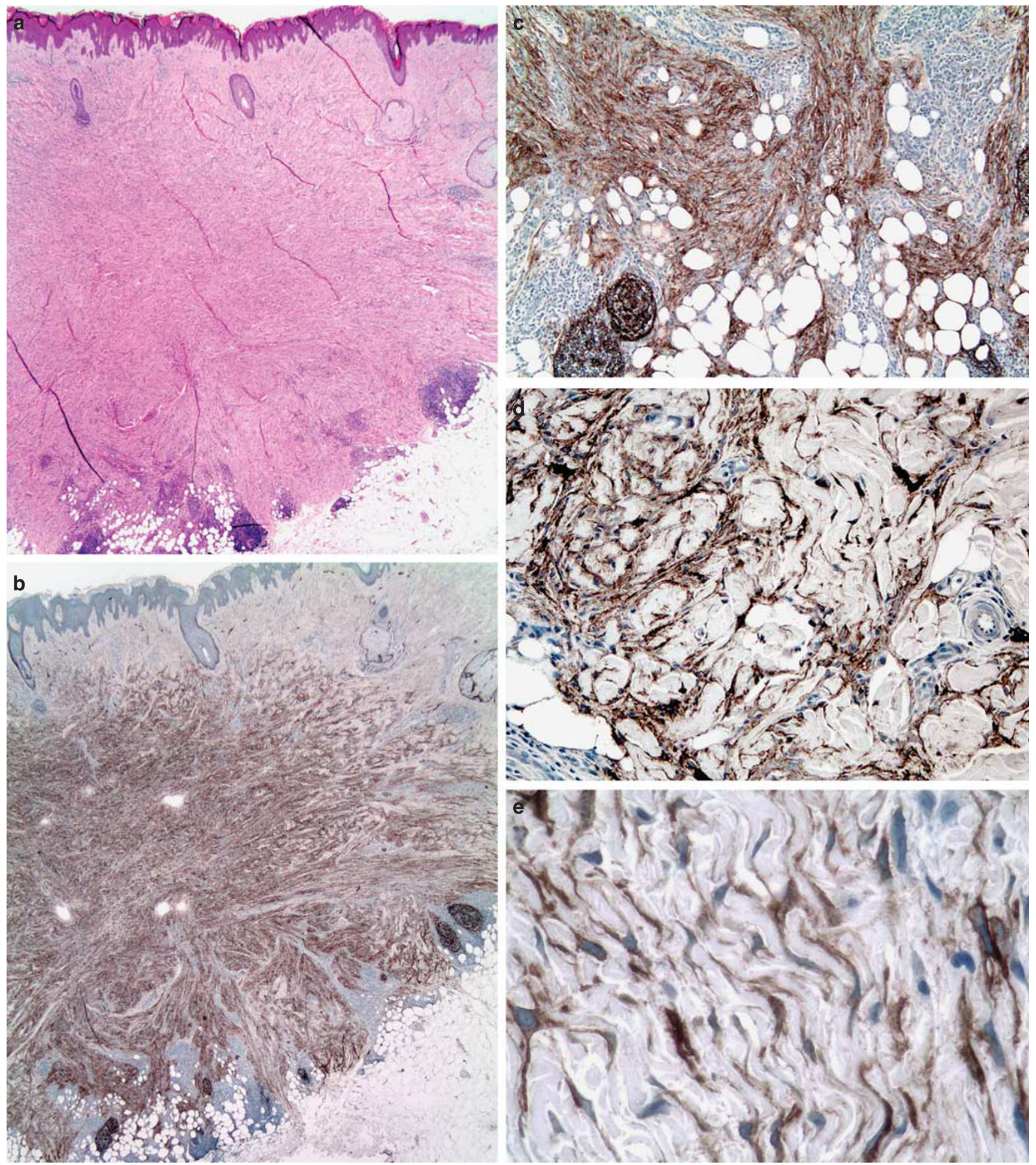

Figure 1 (a) H\&E image of dermatofibroma. (b-e) Dermatofibroma shows strong, diffuse, and crisp cytoplasmic D2-40 positivity with a granular pattern in the spindle cells and follicular dendritic cells in lymphoid aggregates (a and b, $\times 2 ; \mathbf{c}, \times 10 ; \mathbf{d}, \times 40 ; \mathbf{e}, \times 60$ ).

and tumors, including osteocytes and ependymal cells.

To date, there are controversies and uncertainties regarding the histiogenesis of dermatofibromas and dermatofibrosarcoma protuberans. ${ }^{28-31}$ For dermatofibroma, various cells of origin, including fibroblast, histiocyte, and endothelial cell, have been hypothesized. Some authors suggested myofibroblastic derivative for cellular fibrous histiocytoma given its reactivity for $\alpha 1$-smooth muscle actin. On the basis of the characteristic immunoreactivity for factor XIIIa in dermatofibroma, several groups 

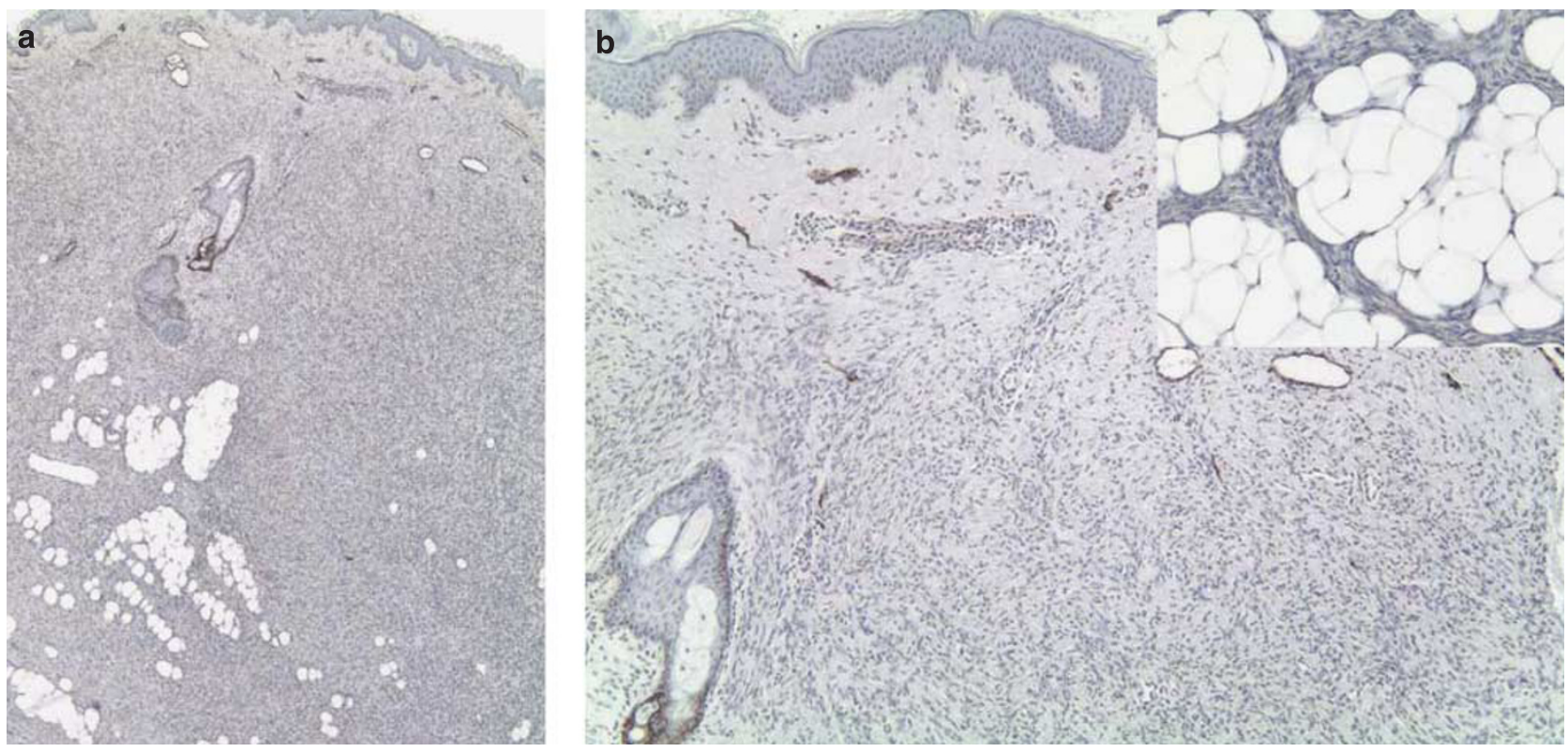

Figure 2 (a, b) Dermatofibrosarcoma protuberans lacks D2-40 staining in the spindle cells. Lymphatic endothelial cells and the basal layer of sebaceous glands are served as positive internal controls $(\mathbf{a}, \times 4 ; \mathbf{b}, \times 10$; insert, $\times 20)$.

postulated an origin from dermal dendrocytes. $^{28,29,32,33}$ Unlike dermatofibroma, earlier immunohistochemical and ultrastructural studies suggested that dermatofibrosarcoma protuberans may be originated from fibroblasts. ${ }^{30,34}$ More recent report indicated that dermatofibrosarcoma protuberans is probably a neuromesenchymal neoplasm based on the observations in Bednar tumors. ${ }^{31}$

In our study, D2-40 was positive in all dermatofibromas, including cellular fibrous histiocytoma. In contrast, none of the dermatofibrosarcoma protuberans was labeled, although four cases showed a patchy and faint background staining. The mechanism of D2-40 immunoreactivity in dermatofibroma is not clear and needs to be investigated. The finding of D2-40 expression in dermatofibroma is interesting given its previously suspected cell of origin as being dermal dendritic cells. Of interest, several recent studies have shown that D2-40 is a highly effective marker for follicular dendritic cells and tumors of this origin. ${ }^{25,35}$ Dendritic cells, including follicular dendritic cells, epidermal Langerhans cells, and dermal dendrocytes, are a heterogeneous group of antigen-presenting cells present in both lymphoid and non-lymphoid tissues. ${ }^{36}$ Although each type differs in its ultrastructure, function, and morphology, dendritic cells from different sites share features of structure and function, such as the ability to capture antigens. Dendritic cells are well known for their motility; for instance, the ones in non-lymphoid organ can migrate to T-dependent areas of lymphoid organs. It is possible that dermatofibroma exhibits D2-40 staining similarly to follicular dendritic cell tumors in light of the dendritic cell commonality. Alternatively, dermal dendritic cells in dermatofibroma may share a common epitope recognizable by D2-40 with follicular dendritic cells, as they are members of the same 'family.' Our data suggest that the cell of origin for dermatofibrosarcoma protuberans is probably not related to dermal dendritic cells due to the lack of D2-40 expression in this tumor.

In summary, we suggest D2-40 utilization as a complementary stain to factor XIIIa and CD34 immunostaining in superficial biopsies to differentiate dermatofibroma and dermatofibrosarcoma protuberans. D2-40 immunostain is helpful in diagnosing and/or confirming the diagnosis of those cases of dermatofibroma and dermatofibrosarcoma protuberans with overlapping features on H\&E stain, CD34, as well as factor XIIIa immunostains.

\section{Disclosure/conflict of interest}

The authors declare no conflict of interest.

\section{References}

1 Calonje E, Mentzel T, Fletcher CD. Cellular benign fibrous histiocytoma. Clinicopathologic analysis of 74 cases of a distinctive variant of cutaneous fibrous histiocytoma with frequent recurrence. Am J Surg Pathol 1994;18:668-676.

2 Vilanova JR, Flint A. The morphological variations of fibrous histiocytomas. J Cutan Pathol 1974;1:155-164.

3 Franquemont DW, Cooper PH, Shmookler BM, et al. Benign fibrous histiocytoma of the skin with potential for local recurrence: a tumor to be distinguished from dermatofibroma. Mod Pathol 1990;3:158-163. 
4 McPeak CJ, Cruz T, Nicastri AD. Dermatofibrosarcoma protuberans: an analysis of 86 cases-five with metastasis. Ann Surg 1967;166:803-816.

5 Fletcher CDM, Evans BJ, Macartney JC, et al. Dermatofibrosarcoma protuberans: a clinicopathological and immunohistochemical study with a review of the literature. Histopathology 1985;9:921-938.

6 Horenstein MG, Prieto VG, Nuckols JD, et al. Indeterminate fibrohistiocytic lesions of the skin. Is there a spectrum between dermatofibroma and dermatofibrosarcoma protuberans? Am J Surg Pathol 2000;24:9961003.

7 Zelger BG, Zelger B. Dermatofibroma. A clinicopathologic classification scheme. Pathologe 1998;19: 412-419.

8 Aiba S, Tabata N, Ishii H, et al. Dermatofibrosarcoma protuberans is a unique fibrohistiocytic tumor expressing CD34. Br J Dermatol 1992;127:79-84.

9 Haycox CL, Odland PB, Olbricht SM, et al. Immunohistochemical characterization of dermatofibrosarcoma protuberance with practical application for diagnosis and treatment. J Am Acad Dermatol 1997;37:438-444.

10 Kutzner H. Expression of human progenitor cell antigen CD34 (HPCA-I) distinguishes dermatofibrosarcoma protuberans from fibrous histiocytoma in formalin-fixed, paraffin-embedded tissue. J Am Acad Dermatol 1993;28:613-617.

11 Cohen PR, Rapini RP, Farhood AI. Dermatofibroma and dermatofibrosarcoma protuberans: differential expression of CD34 and factor XIIIa. Am J Dermatopathol 1994;16:573-574.

12 Goldblum JR, Tuthill RJ. CD34 and factor XIIIa immunoreactivity in dermatofibrosarcoma protuberans and dermatofibroma. Am J Dermatopathol 1997;19: 147-153.

13 Kahn HJ, Fekete E, From L. Tenascin differentiates dermatofibroma from dermatofibrosarcoma protuberans: comparison with CD34 and factor XIIIa. Hum Pathol 2001;32:50-56.

14 Marks A, Sutherland DR, Bailey D, et al. Characterization and distribution of an oncofetal antigen (M2A antigen) expressed on testicular germ cell tumors. Br J Cancer 1999;80:569-578.

15 Hirakawa S, Hong YK, Harvey N, et al. Identification of vascular lineage-specific genes by transcriptional profiling of isolated blood vascular and lymphatic endothelial cells. Am J Pathol 2003;162: $575-586$.

16 Fakunaga M. Expression of D2-40 in lymphatic endothelium of normal tissue and in vascular tumors. Histopathology 2005;46:396-402.

17 Kahn HJ, Bailey D, Marks A. Monoclonal antibody D240, a new marker of lymphatic endothelium, reacts with Kaposi's sarcoma and a subset of angiosarcomas. Mod Pathol 2002;15:434-440.

18 Roy S, Chu A, Trojanowski JQ, et al. D2-40, a novel monoclonal antibody against the M2A antigen as a marker to distinguish hemangioblastoma form renal cell carcinoma. Acta Neuropathol (Berl) 2005;109: 497-502.

19 Galambos C, Nodit L. Identification of lymphatic endothelium in pediatric vascular tumors and malformations. Pediatr Dev Pathol 2005;8:181-189.
20 Debelenko LV, Perez-Atayde AR, Mulliken JB, et al. D2-40 immunohistochemical analysis of pediatric vascular tumors reveal positivity in Kaposiform hemangioendotheliomas. Mod Pathol 2005;18:1454-1460.

21 Rabban JT, Chen Y. D2-40 expression by breast myoepithelium: potential pitfalls in distinguishing intralymphatic carcinoma from in situ carcinoma. Hum Pathol 2008;39:175-183.

22 Gomaa A, Yaar M, Bhawan J. Cutaneous immunoreactivity of D2-40 antibody beyond the lymphatics. Am J Dermatopathol 2007;29:18-21.

23 Browling L, Parker A, Bailey D. D2-40 is a sensitive and specific marker in differentiating primary adrenal cortical tumors from both metastatic clear cell renal cell carcinoma and pheochromocytoma. J Clin Pathol 2008;61:293-296.

$24 \mathrm{Yu} \mathrm{H}$, Gibson JA, Pinkus GS, et al. Podoplanin (D2-40) is a novel marker for follicular dendritic cell tumor. Am J Clin Pathol 2007;128:776-782.

25 Ordonez NG. D2-40 and podoplanin are highly specific and sensitive immunohistochemical markers of epitheloid malignant melanoma. Hum Pathol 2005;36: 372-380.

26 Lau SK, Weiss LM, Chu PG. D2-40 immunohistochemistry in the differential diagnosis of seminoma and embryonal carcinoma: a comparative immunohistochemical study with KIT (VD117) and CD30. Mod Pathol 2007;20:320-325.

27 Schacht V, Dadras SS, Johnson LA, et al. Up-regulation of the lymphatic marker podoplanin, a mucin-type transmembrane glycoprotein, in human squamous cell carcinoma and germ cell tumors. Am J Pathol 2005;166:913-921.

28 Cerio R, Spaull J, Wilson Jones E. Histiocytoma Cutis: a tumor of dermal dendrocytes (dermal dendrocytoma). Br J Dermatol 1989;120:197-206.

29 Cerio R, Spaull J, Oliver GF, et al. A study of factor IIIa and MAC387 immunolabeling in normal and pathological skin. Am J Dermatopathol 1990;12:221-233.

30 Allan AE, Tsou HC, Harrington A. Clonal origin of dermatofibrosarcoma protuberans. Invest Dermatol 1993;100:99-102.

31 Goncharuk V, Mulvaney M, Carlson JA. Bednár tumor associated with dermal melanocytosis: melanocytic colonization or neuroectodermal multidirectional differentiation? J Cutan Pathol 2003;30:147-151.

32 Nestle FO, Nickoloff BJ, Burg G. Dermatofibroma, an abortive immunoreactive process mediated by dermal dendritic cells? Dermatology 1995;190:265-268.

33 Aiba S, Tagami H. Phorbol 12-myristate 13-acetate can transform monocytes-derived dendritic cells to different cell types similar to those found in dermatofibroma. J Cutan Pathol 1998;25:65-71.

34 Dominguez-Malagon H, Valdez-Carrillo Mdel C, CanoValdez AM. Dermatofibroma and dermatofibrosarcoma protuberance: a comprehensive ultrastructural study. Ultrastructural Pathol 2006;30:283-291.

35 Marsee DK, Pinkus GS, Hornick JL. Podoplanin (D2-40) is a highly effective marker of follicular dendritic cells. Appl Immunohistochem Mol Morphol 2009;17:102-107.

36 Steinman RM. The dendritic cell system and its role in immunogenicity. Annu Rev Immunol 1991;9:271-296. 